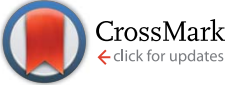

Cite this: RSC Adv., 2017, 7, 7041

\title{
Tetroxime: reactivation potency - in vitro and in silico study
}

\author{
K. Kuca, ${ }^{\text {abc }}$ J. Korabecny, ${ }^{\text {ad }}$ R. Dolezal, ${ }^{a}$ E. Nepovimova, ${ }^{\text {ad }}$ O. Soukup, ${ }^{a}$ L. Gorecki $^{\text {ad }}$ \\ and K. Musilek ${ }^{\mathrm{abd}}$
}

Acetylcholinesterase (AChE) reactivators (oximes) are generally used as treatment in cases of nerve agent poisoning. There is no single oxime applicable in every case of nerve agent intoxication. Based on this fact, novel candidates with broader efficacy are still being sought. In this study, tetroxime a bisquaternary compound bearing four oxime groups, was evaluated for its potency to reactivate rat brain AChE inhibited by selected nerve agents (tabun, sarin, cyclosarin and VX agent). Despite the fact that this oxime contains four oxime groups that could be plausibly responsible for reactivation, it did not achieve broader reactivation activity. Satisfactory results were obtained only in the case of VX agent-inhibited AChE. In the cases of sarin- and cyclosarin-inhibited AChE, acceptable results were reached at higher oxime concentration only. Tetroxime was unable to reactivate tabun-inhibited AChE. However, compared with the gold standard pralidoxime, this oxime achieved more promising results.

Received 26th June 2016

Accepted 14th November 2016

DOI: 10.1039/c6ra16499d

www.rsc.org/advances

\section{Introduction}

Nerve agents such as sarin, tabun or VX agent are currently considered to be a potential terrorist threat because of their extraordinarily high acute toxicity at relatively low doses. ${ }^{1}$ In the past, these compounds have been misused by terrorists - in the Tokyo sarin subway attack (1995), or in local conflicts such as the Iran-Iraq War. ${ }^{2,3}$ Quite recently, sarin has been used against civilians in Syria. ${ }^{4}$ Although these chemical warfare agents have been known for decades, no universal antidote has been developed so far to counteract nerve agent poisoning. ${ }^{5,6}$

From the biochemical point of view, nerve agents covalently bind to serine in the catalytic active site of acetylcholinesterase (AChE; EC 3.1.1.7), forming a P-O covalent bond. Afterwards, the inhibited enzyme is unable to degrade the neuromediator acetylcholine (ACh) at the synaptic cleft. Thereafter, ACh accumulates at overstimulated nicotinic and muscarinic receptors resulting in severe cholinergic crisis. Without immediate treatment, the intoxicated organism dies as a consequence of respiratory failure. ${ }^{7}$

Anticholinergics such as atropine and AChE reactivators are generally used as antidotal therapy in cases of intoxication

${ }^{a}$ Biomedical Research Center, University Hospital Hradec Kralove, Czech Republic. E-mail: kamil.kuca@uhk.cz

${ }^{b}$ Department of Chemistry, Faculty of Science, University of Hradec Kralove, Hradec Kralove, Czech Republic

'Department of Cellular Biology \& Pharmacology, Herbert Wertheim College of Medicine, Florida International University, Miami, Florida, USA

${ }^{d}$ Department of Toxicology and Military Pharmacy, Faculty of Military Health Sciences, University of Defence, Hradec Kralove, Czech Republic with nerve agent or organophosphorus pesticide poisoning. ${ }^{8}$ Currently, the most promising reactivator - oxime HI-6 - shows promising reactivation potency in cases of sarin, cyclosarin or VX-agent poisoning, ${ }^{9,10}$ but has no reactivation effect against tabun or organophosphorus pesticide poisonings. ${ }^{11-13}$ Unfortunately, this oxime is currently not commercially available in many countries. Obidoxime seems to be the most widely used oxime. It is generally used for treatment of nerve agent and organophosphorus pesticide poisonings mainly in European countries. ${ }^{14}$ However, its main limitations are its high toxicity and its low reactivation potency in the case of cyclosarin. ${ }^{15,16}$ Pralidoxime is another well-known reactivator, but it is already an obsolete compound with low reactivation potency against both nerve agents and oraganophosphorus pesticide poisoning. ${ }^{17-20}$

Against this background, research and development on novel oxime candidates still continues. Several novel AChE reactivators with improved reactivation potency have been developed. ${ }^{5,21}$ However, none of these reactivators possesses broad-spectrum reactivation potency. ${ }^{22,23}$

In this contribution, tetroxime - a unique bisquaternary compound with four oxime groups was tested in order to find out if four nucleophilic groups could increase the reactivation activity (Fig. 1). For this purpose a standard in vitro reactivation assay was used..$^{24,25}$ Moreover, a docking study was undertaken to reveal a plausible molecular setting for tetroxime for reactivation in the cavity of AChE inhibited by VX-agent. Tabun, sarin, cyclosarin and VX agents were selected as main members of the nerve agent family. Pralidoxime (2-PAM, Fig. 1), a gold standard, was used for comparison. 
<smiles>O=Nc1cc[n+](COC[n+]2ccc(C=NO)cc2C=NO)c(C=NO)c1</smiles>

tetroxime

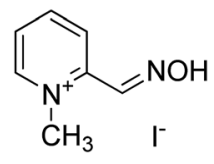

2-PAM

Fig. 1 Chemical structures of tetroxime and pralidoxime.

\section{Material \& methods}

\section{In vitro experiment}

Chemicals. Tetroxime (chemical name: 1-[(\{2,4-bis[(1E)(hydroxyimino)methyl]pyridin-1-ium-1-yl\}methoxy)methyl]-2[(1E)-(hydroxyimino)methyl]-4-[(1Z)-(hydroxyimino)methyl]pyridin-1-ium dibromide) used in this in vitro study was synthesized in our laboratory prior to the experiment using the standard synthetic approach. ${ }^{16}$ The purity of this oxime reactivator was controlled using TLC and HPLC methods developed earlier. ${ }^{26,27}$ Nerve agents - tabun, sarin, cyclosarin and VX agent were obtained from Military Technical Institute (Brno, Czech Republic) and were of $95 \%$ purity. All other chemicals used in this experiment were purchased from Sigma-Aldrich (Czech Republic) and were of analytical purity.

Enzyme sources. Rat brain homogenate $(10 \%$ in distilled water) was used as a source of AChE. Following standard European bioethical directives, Wistar rats $(n=6$, BioTest Konarovice, Czech Republic) were first narcotized and then decapitated. Afterwards, their brains were isolated and homogenized (IKA Ultra-Turrax homogenizer, Sigma Aldrich, Czech Republic) in distilled water to obtain $10 \%$ homogenate. The experiment was performed in compliance with Czech legislation and institutional guidelines of the Faculty of Military Health Sciences, University of Defence. The institutional ethical committee of the Faculty of Military Health Sciences, University of Defence approved the experiment.

In vitro method. The reactivation activity of tetroxime was tested in vitro on nerve agent-inhibited AChE using slightly modified standard reactivation test. ${ }^{24,25}$ Brain homogenate (0.5 $\mathrm{mL}$ ) was mixed with isopropanol solution of the selected nerve agent (amount of the nerve agent causing 95\% inhibition of AChE) and incubated at $25{ }^{\circ} \mathrm{C}$ for $30 \mathrm{~min} .2 .5 \mathrm{~mL}$ of sodium chloride solution $(3 \mathrm{M})$ were added to the mixture and made up

to a volume of $23 \mathrm{~mL}$ with distilled water. Finally, $2 \mathrm{~mL}$ of acetylcholine iodide solution $(0.02 \mathrm{M})$ were added. Enzyme activity was measured at $\mathrm{pH} 8.0$ and temperature $25^{\circ} \mathrm{C}$ using an autotitrator RTS 822 (Radiometer, Denmark). The activities of intact $\operatorname{AChE}\left(a_{0}\right)$ and inhibited AChE $\left(a_{\mathrm{i}}\right)$ were derived from the influence of consumption of $\mathrm{NaOH}$ solution $(0.01 \mathrm{M})$ with time. After the incubation of AChE with nerve agent $(30 \mathrm{~min})$, tetroxime was added to the solution and the mixture was incubated for $10 \mathrm{~min}$. The activity of reactivated AChE $\left(a_{\mathrm{r}}\right)$ was also derived from the influence of consumption of $\mathrm{NaOH}$ solution with time.

The percentage of reactivation (\%) was calculated from the measured data according to the equation:

$$
x=\left(1-\frac{a_{\mathrm{r}}-a_{\mathrm{i}}}{a_{0}-a_{\mathrm{i}}}\right) \times 100[\%]
$$

Each measurement was repeated three times. Differences between mean values were analyzed for significance using the Student's unpaired $t$-test. The difference was considered as significant when $P$ value was less than 0.05 .

\section{Molecular docking studies}

From the online RCSB protein databank (http://www.pdb.org) a model of VX-inhibited mAChE (PDB ID: 2Y2U, resolution: 2.60 A) was downloaded and prepared for flexible molecular docking by MGL Tools utilities. The preparation of this receptor involved removal of the second chain B, addition of polar hydrogens, and merging of non-polar ones. Default gasteiger charges were assigned to all atoms. Flexible parts of the enzyme were determined by a spherical selection of residues $(R=11 \AA)$ around the phosphorus atom of VX in the active site of MAChE. The grid box of $33 \times 33 \times 33 \AA$ was positioned at the geometrical center of the selected flexible residues volume. The rotatable bonds in the flexible residues were detected automatically by AutoDock Tools 1.5.4 program. Because of the limitation of the program used for flexible molecular docking, water molecules had to be removed from the system. Flexible receptor parts contained 27 residues for mAChE. The $x y z$ coordinates of the grid box center were applied as mAChE $(28.21,15.53,12.40)$. The studied ligand tetroxime was firstly drawn in HyperChem 8.0, then manually protonated as suggested by MarvinSketch 6.2.0 software (http:// www.chemaxon.com), geometrically optimized by semiempirical quantum-chemistry RM1 method and stored as a pdb file. The structure of the ligand was processed for docking

Table 1 In vitro reactivation activity of tetroxime

\begin{tabular}{|c|c|c|c|c|}
\hline Sarin & 0 & 54 & 4 & 14 \\
\hline Cyclosarin & 0 & 0 & 0 & 20 \\
\hline VX agent & 0 & 34 & 29 & 46 \\
\hline
\end{tabular}




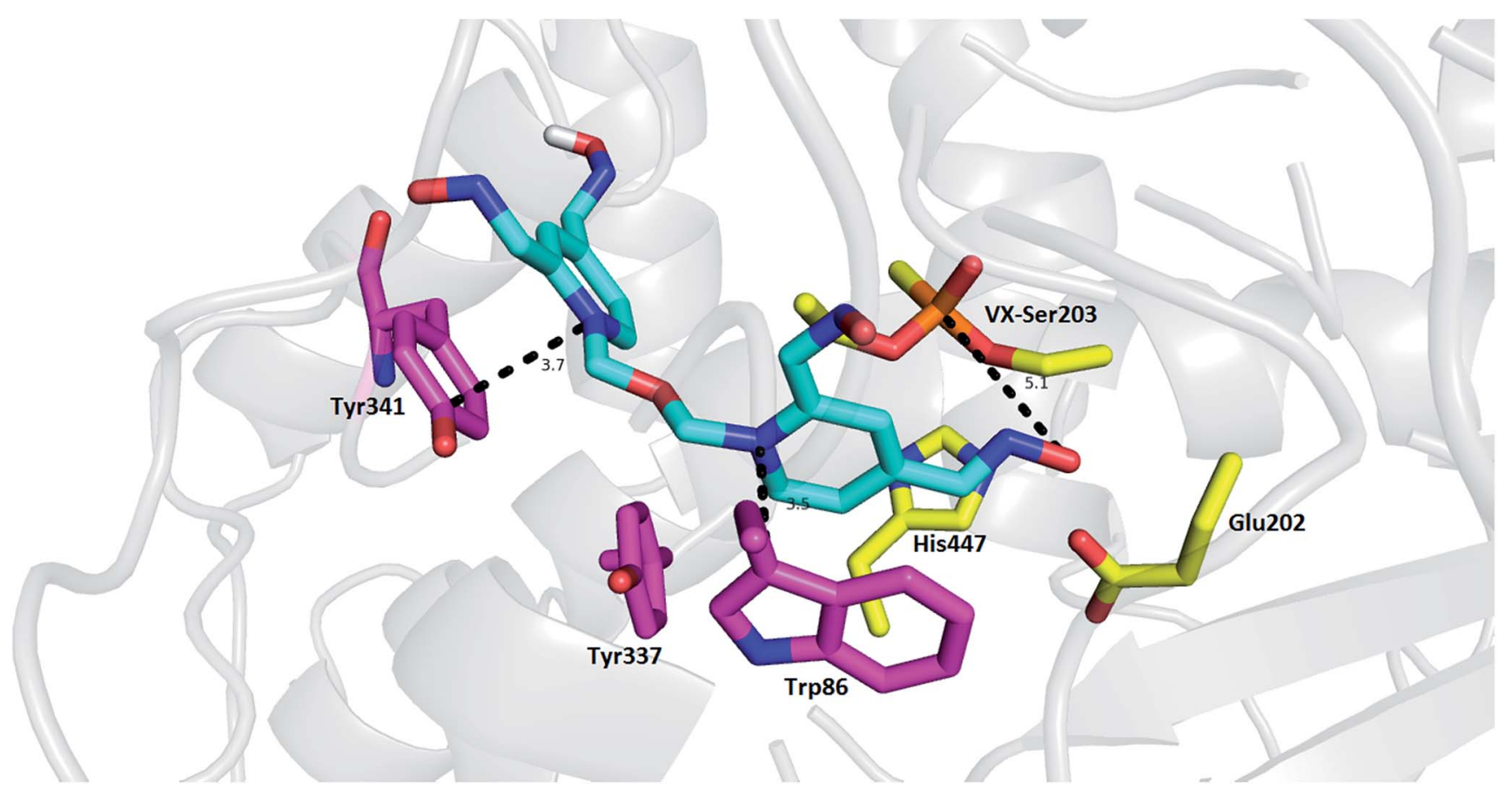

Fig. 2 Top-scored docking pose of tetroxime in the cavity of non-aged VX-inhibited mAChE. Ligand is displayed in blue carbon atoms, catalytic triad residues in yellow and other relevant amino acids involved in ligand-enzyme interaction in purple. The rest of the enzyme is shown in grey cartoon. Figure was created with PyMol 1.30.

in a similar way as the above-mentioned flexible parts of the receptor by AutoDock Tools 1.5.4 program. Molecular docking was carried out in AutoDock Vina 1.1.2 program utilizing computer resources of the Czech National Grid Infrastructure MetaCentrum. Regarding the search algorithm of AutoDock Vina, it efficiently combines Markov chain Monte Carlo-like method for the global search and Broyden-Fletcher-GoldfarbShano gradient approach for the local search. It is a type of memetic algorithm based on interleaving stochastic and deterministic calculations. Each docking task was repeated 10 times with the exhaustiveness parameter set to 16, employing 16 CPUs multithreading in parallel. From the obtained results, solutions achieving the minimum of predicted Gibbs binding energy were taken as the top-scoring mode. The graphic representations of the docked poses were rendered in PyMOL 1.3 (The PyMOL Molecular Graphics System, Version 1.5.0.4 Schrödinger, LLC.).

\section{Results and discussion}

Recently, much effort has been invested worldwide to develop novel oximes. ${ }^{1,21,31,32}$ Although a plethora of novel drug candidates has been prepared, a novel broad-spectrum oxime is still lacking. ${ }^{6,23}$ As mentioned before, oxime HI- 6 could not become a universal reactivator with a broad spectrum of reactivating potency because of its deficiency in treatment of tabun and organophosphorus pesticide poisoning. ${ }^{9-13}$ HLö-7, which gained much attention in terms of interest and perspective, also has several limitations (poor tabun reactivation; synthetic feasibility, etc.). ${ }^{33}$ Neither novel oximes from the K-series could be considered as broad spectrum oximes..$^{11,19,24}$ In light of this, development of novel oximes and testing of old ones continues.

Several groups working in the "oxime area" are focused more to the civil sector - to oximes applicable in cases of organophosphorus pesticide poisoning. ${ }^{34,35}$ It is generally accepted that oximes originally developed for the treatment of nerve agent poisoning are not good antidotes for pesticide poisoning. Thus, novel oximes have been designed specifically for pesticides. ${ }^{13,18}$ There are other laboratories which do not have permission to work with "real" nerve agents, thus these laboratories use OP pesticides as mimics of nerve agents..$^{17,19,20}$

Several promising oximes recently underwent more sophisticated studies such as pharmacokinetics or genotoxicity, as a necessary preclinical prelude before administration to humans within clinical trials..$^{34,35}$

Results obtained in this study are summarized in Table 1. Satisfactory reactivation results were obtained in the case of VX agent-inhibited AChE only. In the cases of sarin- and cyclosarininhibited AChE, moderate results were reached only at higher oxime concentration. However, such concentration is not attainable after administration of relevant human doses. ${ }^{8}$ Tetroxime was unable to reactivate tabun-inhibited AChE.

Unfortunately, pralidoxime did not reach any reactivation efficacy at relevant human doses. In higher concentration, it was able to reactivate sarin and VX agent-inhibited AChE.

Tetroxime mechanism of action was explored using molecular modeling studies in order to gain insight into determinants responsible for the reactivation process. As no structure model for the rat OP-AChE complex was available, we used a model of mouse AChE (mAChE, PDB ID: 2Y2U) instead because of the 
high degree of interspecies similarity with AChE. ${ }^{28,29}$ As depicted in Fig. 2, the top-scored docking pose of tetroxime is wellaccommodated in the VX-inhibited mAChE active site. The proximal pyridinium moiety is stabilized by $\pi-\pi /$ cation $-\pi$ interactions with Trp86 (3.5 $\AA$ ) in the catalytic anionic site. In similar fashion, the distal pyridinium ring protrudes out of the gorge providing parallel $\pi-\pi /$ cation $-\pi$ contact to Tyr341 (3.7 $⿱$ A). As suggested by MarvinSketch protonation under the conditions used in in vitro assessment $(\mathrm{pH}=8.0)$, we can suggest that the oximate responsible for cleavage of the $\mathrm{O}-\mathrm{P}$ bond can be formed at position 2 of the pyridinium ring (88\%), and only $6 \%$ is predicted for the oximate at position 4 . However, the oxime group at position 4- of the proximal pyridinium ring resided in a more favorable position to cleave the O-P bond, although further molecular rearrangement would have to come about to form the transition state with the bond angle $\mathrm{O}(\mathrm{Ser})-\mathrm{P}(\mathrm{VX})-$ $\mathrm{O}$ (oximate) settled at approximately $180^{\circ} .^{30}$ According to additional quantum chemistry simulation by the semi-empirical method RM1 in HyperChem, the equilibrium $\mathrm{P}(\mathrm{VX})-\mathrm{O}(\mathrm{oxi}-$ mate) bond length of the transition state in vacuum is about 1.9 A. Accordingly far-from-optimal distance formed between oximate and phosphorus atom resulting from molecular docking is $5.1 \AA$. Taken together, based on obtained data from in silico and contrary findings from in vitro, such orientation in $\mathrm{mAChE}$ suggests that tetroxime is not a convenient reactivator for organophosphorus poisoning, even using the model of nonaged VX-inhibited mAChE. These data might also point out to low reactivation potency related to other nerve agents used in in vitro studies disclosing that more oxime groups rather confer detrimental effect in terms of steric hindrance and electron distribution in the ligand.

\section{Conclusions}

In this study, tetroxime - a reactivator bearing four oxime groups - was tested in vitro. Only limited reactivation potency was found, and in the case of tabun no reactivation was found under the experimental conditions. The reactivation efficacy was also explored using docking studies. In silico observations are fully consistent with data obtained in vitro.

In general, based on these findings, tetroxime could not be recommended for further studies (preclinical trials). However, the results obtained here should be of interest to those focused on structure-activity studies, in that increasing the number of oxime groups in a single molecule does not necessarily increase the oxime reactivation potency.

\section{Acknowledgements}

The work was supported by University of Defence (Long Term Development Plan-1011, SV/FVZ201601), by MH CZ-DRO (University Hospital Hradec Kralove, No. 00179906), by the Czech Science Foundation (No. GA15-16701S) and University of Hradec Kralove (Faculty of Science, No. SV2110/2016). The authors are grateful to Ian McColl MD, PhD for assistance with the manuscript.

\section{Notes and references}

1 K. Musilek, M. Dolezal, F. Gunn-Moore and K. Kuca, Med. Res. Rev., 2011, 31, 548-575.

2 A. T. Tu, Forensic Sci. Rev., 2014, 26, 115-120.

3 D. D. Haines and S. C. Fox, Forensic Sci. Rev., 2014, 26, 97114.

4 Y. Rosman, A. Eisenkraft, N. Milk, A. Shiyovich, N. Ophir, S. Shrot, Y. Kreiss and M. Kassirer, Ann. Intern. Med., 2014, 160, 644-648.

5 J. Korabecny, O. Soukup, R. Dolezal, K. Spilovska, E. Nepovimova, M. Andrs, T. D. Nguyen, D. Jun, K. Musilek, M. Kucerova-Chlupacova and K. Kuca, MiniRev. Med. Chem., 2014, 14, 215-221.

6 K. Kuca, D. Jun and J. Bajgar, Drug Chem. Toxicol., 2007, 30, 31-40.

7 T. C. Marrs, P. Rice and J. A. Vale, Toxicol. Rev., 2006, 25, 297323.

8 J. Bajgar, J. Fusek, K. Kuca, L. Bartosova and D. Jun, Mini-Rev. Med. Chem., 2007, 7, 461-466.

9 P. M. Lundy, L. Raveh and G. Amitai, Toxicol. Rev., 2006, 25, 231-243.

10 P. M. Lundy, M. G. Hamilton, T. W. Sawyer and J. Mikler, Toxicology, 2011, 285, 90-96.

11 J. Kassa, K. Kuca, J. Cabal and M. Paar, J. Toxicol. Environ. Health, Part A, 2006, 69, 1875-1882.

12 A. Lucić Vrdoljak, M. Calić, B. Radić, S. Berend, D. Jun, K. Kuca and Z. Kovarik, Toxicology, 2006, 228, 41-50.

13 K. Musilek, O. Holas, K. Kuca, D. Jun, V. Dohnal, V. Opletalova and M. Dolezal, Bioorg. Med. Chem. Lett., 2007, 17, 3172-3176.

14 D. Steinritz, F. Eyer, F. Worek, H. Thiermann and H. John, Toxicol. Lett., 2016, 244, 121-123.

15 K. Kuca and J. Patocka, J. Enzyme Inhib. Med. Chem., 2004, 19, 39-43.

16 K. Musilek, O. Holas, J. Hambalek, K. Kuca, D. Jun, V. Dohnal and M. Dolezal, Lett. Org. Chem., 2006, 3, 831-835.

17 G. A. Petroianu, K. Arafat, S. M. Nurulain, K. Kuca and J. Kassa, J. Appl. Toxicol., 2007, 27, 168-175.

18 K. Musilek, O. Holas, K. Kuca, D. Jun, V. Dohnal and M. Dolezal, J. Enzyme Inhib. Med. Chem., 2007, 22, 425-432.

19 D. E. Lorke, M. Y. Hasan, K. Arafat, K. Kuca, K. Musilek, A. Schmitt and G. A. Petroianu, J. Appl. Toxicol., 2008, 28, 422-429.

20 S. M. Nurulain, D. E. Lorke, M. Y. Hasan, M. Shafiullah, K. Kuca, K. Musilek and G. A. Petroianu, Neurotoxic. Res., 2009, 16, 60-67.

21 G. Mercey, T. Verdelet, J. Renou, M. Kliachyna, R. Baati, F. Nachon, L. Jean and P.-Y. Renard, Acc. Chem. Res., 2012, 45, 756-766.

22 M. Winter, T. Wille, K. Musilek, K. Kuca, H. Thiermann and F. Worek, Toxicol. Lett., 2016, 244, 136-142.

23 F. Worek, J. von der Wellen, K. Musilek, K. Kuca and H. Thiermann, Arch. Toxicol., 2012, 86, 1379-1386.

24 K. Kuca and J. Kassa, J. Enzyme Inhib. Med. Chem., 2003, 18, 529-535. 
25 K. Kuca and J. Cabal, Toxicol. Mech. Methods, 2005, 15, 247252.

26 D. Jun, P. Stodulka, K. Kuca, V. Koleckar, B. Dolezal, P. Simon and M. Veverka, J. Chromatogr. Sci., 2008, 46, 316-319.

27 D. Jun, P. Stodulka, K. Kuca and B. Dolezal, J. Chromatogr. Sci., 2010, 48, 694-696.

28 J. Wiesner, Z. Kriz, K. Kuca, D. Jun and J. Koca, J. Enzyme Inhib. Med. Chem., 2007, 22, 417-424.

29 R. Dolezal, J. Korabecny, D. Malinak, J. Honegr, K. Musilek and K. Kuca, J. Mol. Graphics Modell., 2015, 56, 113-129.

30 L. Wong, Z. Radic, R. J. Brüggemann, N. Hosea, H. A. Berman and P. Taylor, Biochemistry, 2000, 39, 5750-5757.

31 E. Nepovimova, J. Korabecny, R. Dolezal, T. D. Nguyen, D. Jun, O. Soukup, M. Pasdiorova, P. Jost, L. Muckova,
D. Malinak, L. Gorecki, K. Musilek and K. Kuca, Toxicol. Res., 2016, 5, 1012-1016.

32 L. Gorecki, J. Korabecny, K. Musilek, D. Malinak, E. Nepovimova, R. Dolezal, D. Jun, O. Soukup and K. Kuca, Arch. Toxicol., 2016, 90, 2831-2859.

33 K. Kuca, K. Musilek, J. Karasova, D. Jun, O. Soukup, M. Pohanka, K. K. Ghosh and M. Hrabinova, Mini-Rev. Med. Chem., 2011, 80-84.

34 H. Kalász, M. Y. Hasan, R. Sheen, K. Kuca, G. Petroianu, K. Ludányi, A. Gergely and K. Tekes, Anal. Bioanal. Chem., 2006, 385, 1062-1067.

35 K. Tekes, M. Y. Hasan, R. Sheen, K. Kuca, G. Petroianu, K. Ludányi and H. Kalász, J. Chromatogr. A, 2006, 1122, 8487. 